Portland State University

PDXScholar

Electrical and Computer Engineering Faculty

Publications and Presentations

$12-1-1997$

\title{
Sinusoidal-Gaussian Beams in Complex Optical Systems
}

Lee W. Casperson

Portland State University

Dennis G. Hall

University of Rochester

Anthony A. Tovar

Murray State University

Follow this and additional works at: https://pdxscholar.library.pdx.edu/ece_fac

Part of the Electrical and Computer Engineering Commons

Let us know how access to this document benefits you.

\section{Citation Details}

Lee W. Casperson, Dennis G. Hall, and Anthony A. Tovar, "Sinusoidal-Gaussian beams in complex optical systems," J. Opt. Soc. Am. A 14, 3341-3348 (1997).

This Article is brought to you for free and open access. It has been accepted for inclusion in Electrical and Computer Engineering Faculty Publications and Presentations by an authorized administrator of PDXScholar. Please contact us if we can make this document more accessible: pdxscholar@pdx.edu. 


\title{
Sinusoidall-Gaussian beams in complex optical systems
}

\author{
Lee W. Casperson* and Dennis G. Hall \\ The Institute of Optics and The Rochester Theory Center for Optical Science and Engineering, \\ University of Rochester, Rochester, New York 14627-0186
}

\author{
Anthony A. Tovar \\ Department of Physics and Engineering Physics, Murray State University, Murray, Kentucky 42071-0009
}

Received February 12, 1997; revised manuscript received May 27, 1997; accepted June 13, 1997

\begin{abstract}
Sinusoidal-Gaussian beam solutions are derived for the propagation of electromagnetic waves in free space and in media having at most quadratic transverse variations of the index of refraction and the gain or loss. The resulting expressions are also valid for propagation through other real and complex lens elements and systems that can be represented in terms of complex beam matrices. The solutions are in the form of sinusoidal functions of complex argument times a conventional Gaussian beam factor. In the limit of large Gaussian beam size, the sine and cosine factors of the beams are dominant and reduce to the conventional modes of a rectangular waveguide. In the opposite limit the beams reduce to the familiar fundamental Gaussian form. Alternate hyperbolic-sinusoidal-Gaussian beam solutions are also found. (C) 1997 Optical Society of America [S0740-3232(97)00212-3]
\end{abstract}

\section{INTRODUCTION}

One of the most basic problems in optics is the determination of the propagation characteristics of electromagnetic beam waves in various optical elements and systems. Historically, the simplest limit of propagation in free space was the first to be analyzed in detail, and it was found that the field solutions in that case can be described in terms of Laguerre-Gaussian functions of real argument (referring to the argument of the Laguerre function) if one is working in cylindrical coordinates ${ }^{1}$ or Hermite-Gaussian functions of real argument in rectangular coordinates. ${ }^{2}$ However, many optical elements of interest involve spatial variations of the index of refraction, gain, or loss; and it is also important to understand the propagation characteristics of optical beams in such elements. It has long been known that media with refraction profiles can be used to guide electromagnetic beams. The guiding of beams by media with gain or loss profiles was also predicted, ${ }^{3}$ and gain guiding has been demonstrated experimentally. ${ }^{4}$ However, the propagation of higher-order electromagnetic beams in such complex media cannot be easily described in terms of polynomial-Gaussian solutions of real argument. Complex Hermite-Gaussian beams ${ }^{5}$ and Laguerre-Gaussian beams ${ }^{6}$ were discovered as eigenfunctions for laser resonators with Gaussian mirror-reflectivity profiles, and the complex Hermite-Gaussian beams were also found in more general propagation studies. ${ }^{7-16}$ In particular, it was shown that complex off-axis Hermite-Gaussian and Laguerre-Gaussian beams can propagate in any medium that can be characterized by only constant, linear, and quadratic transverse variations of the gain and index of refraction in the vicinity of the beam. ${ }^{11,12,16}$ Thus such beams are needed for the most general analytic propagation studies.

There are important qualitative distinctions between the propagation behavior of polynomial-Gaussian beams of real argument and the behavior of the corresponding beam solutions of complex argument. With the realargument solutions the polynomial-Gaussian fields retain the general form of their field distribution at all planes along the propagation path. Thus the scale of the amplitude profile may change, but its general shape is constant. With the complex beams, on the other hand, the field profile may change form dramatically from one plane to another. A further important difference is that the spatial phase variations of the real-argument beams are similar to spherical waves, whereas the complexargument beams have more complicated phase profiles.

Although most attention over the years has been focused on Hermite-Gaussian and Laguerre-Gaussian field solution sets, other solutions are possible, too. It was shown that linearly polarized $\mathrm{J}_{0}$-Bessel-Gaussian beams can propagate in free space, ${ }^{17,18}$ and recently a set of higher order azimuthally or radially polarized freespace Bessel-Gaussian beams was reported. ${ }^{19,20}$ A set of higher-order linearly polarized beams can also be obtained. Like the polynomial-Gaussian solutions of complex argument, the field distribution of these beams evolves strongly with propagation distance.

An attractive feature of the Bessel-Gaussian beams is their correspondence with the modes of waveguides. Thus in the limit that the Gaussian beam size becomes large, the remaining Bessel function factor corresponds exactly with the Bessel function modes of dielectric waveguides such as optical fibers. ${ }^{21}$ These solutions are 
also the same as the so-called nondiffracting beams of recent interest. ${ }^{22,23}$ Such beams have been called nondiffracting on the basis that, if a sufficient portion of the beam profile is available, the beam will propagate for a long distance without much reduction in the amplitude of the central maximum (or any other maximum).

The new Bessel-Gaussian beams are one of the natural solutions of the wave equation in cylindrical coordinates. It is reasonable to inquire whether there might also be a corresponding set of beams in rectangular coordinates. Such a set should ideally reduce to conventional rectangular waveguide modes in an appropriate limit. One purpose of this work has been to develop a set of sinusoidal-Gaussian field solutions of complex argument for beam propagation in a rectangular system. In the limit that the Gaussian becomes infinitely wide, these solutions reduce to the ordinary sinusoidal modes that one would expect for a rectangular waveguide. Local maxima of such modes are, of course, also nondiffracting.

The derivation of our sinusoidal-Gaussian beams has included the possibility of propagation in complex lenslike media having at most quadratic transverse variations of the index of refraction and the gain or loss. The special case of propagation in free space is also considered. The beams also propagate in any of the optical elements or systems that have previously been analyzed in terms of polynomial-Gaussian functions. Advanced matrix methods for off-axis beams in general misaligned systems are also applicable to this solution set. ${ }^{24}$

The basic derivation of the sinusoidal-Gaussian beams is included in Section 2. The purpose of this derivation is to reduce the partial differential wave equation to a set of first-order ordinary differential equations governing the various parameters that characterize the beam. The solutions of these simpler equations are discussed in Section 3, and the specific problem of propagation through free space is explored in Section 4.

\section{DERIVATION OF THE BEAM SOLUTIONS}

For any investigation of light propagation the proper starting point is the Maxwell-Heaviside equations. These equations can be combined to yield coupled-wave equations that govern the various field components of a propagating electromagnetic beam. For the usual case of slowly varying complex propagation constant $k$, the dominant transverse field components are governed by the much simpler wave equation

$$
\nabla^{2} \mathbf{E}^{\prime}(x, y, z)+k^{2}(x, y, z) \mathbb{E}^{\prime}(x, y, z)=0,
$$

where $\mathbf{E}^{\prime}$ is the complex amplitude of the vector electric field $\mathbf{E}$, and $k$ is the complex spatially dependent wave number. The wave number may have an imaginary part as a result of nonzero conductivity or out-of-phase components of the material polarization or magnetization. If needed, the weak $z$ components of the fields may be found from the transverse components by means of the Maxwell-Heaviside equations. ${ }^{11}$

In many practical situations the gain (or loss) and index of refraction have at most quadratic variations in the vicinity of the propagating beam, and one can write

$$
\begin{aligned}
k^{2}(x, y, z)= & k_{0}(z)\left[k_{0}(z)-k_{1 x}(z) x-k_{1 y}(z) y\right. \\
& \left.-k_{2 x}(z) x^{2}-k_{2 y}(z) y^{2}\right] .
\end{aligned}
$$

For an $x$-polarized wave propagating in the $z$ direction a useful substitution is

$$
\mathbb{E}_{x}{ }^{\prime}(x, y, z)=A(x, y, z) \exp \left[-i \int_{0}^{z} k_{0}\left(z^{\prime}\right) \mathrm{d} z^{\prime}\right],
$$

and the $x$ component of Eq. (1) reduces to

$$
\begin{aligned}
\frac{\partial^{2} A}{\partial x^{2}}+\frac{\partial^{2} A}{\partial y^{2}}-2 i k_{0} \frac{\partial A}{\partial z}-i & \frac{\mathrm{d} k_{0}}{\mathrm{~d} z} A-k_{0}\left(k_{1 x} x+k_{1 y} y\right. \\
& \left.+k_{2 x} x^{2}+k_{2 y} y^{2}\right) A=0, \quad \text { (4) }
\end{aligned}
$$

where $A(x, y, z)$ is assumed to vary so slowly with $z$ that its second derivative can be neglected.

A useful form for an astigmatic off-axis Gaussian beam is $^{12}$

$$
\begin{aligned}
A(x, y, z)= & B(x, y, z) \exp \left\{-i\left[\frac{Q_{x}(z) x^{2}}{2}+\frac{Q_{y}(z) y^{2}}{2}\right.\right. \\
& \left.\left.+S_{x}(z) x+S_{y}(z) y\right]\right\}
\end{aligned}
$$

With this substitution Eq. (4) may be separated into the set

$$
\begin{aligned}
& Q_{x}^{2}+k_{0} \frac{\mathrm{d} Q_{x}}{\mathrm{~d} z}+k_{0} k_{2 x}=0, \\
& Q_{y}{ }^{2}+k_{0} \frac{\mathrm{d} Q_{y}}{\mathrm{~d} z}+k_{0} k_{2 y}=0 \\
& Q_{x} S_{x}+k_{0} \frac{\mathrm{d} S_{x}}{\mathrm{~d} z}+\frac{k_{0} k_{1 x}}{2}=0 \\
& Q_{y} S_{y}+k_{0} \frac{\mathrm{d} S_{y}}{\mathrm{~d} z}+\frac{k_{0} k_{1 y}}{2}=0, \\
& \frac{\partial^{2} B}{\partial x^{2}}-2 i\left(S_{x}+Q_{x} x\right) \frac{\partial B}{\partial x}+\frac{\partial^{2} B}{\partial y^{2}}-2 i\left(S_{y}+Q_{y} y\right) \frac{\partial B}{\partial y} \\
& -\left(S_{x}{ }^{2}+S_{y}{ }^{2}\right) B-i\left(Q_{x}+Q_{y}\right) B-2 i k_{0} \frac{\partial B}{\partial z} \\
& -i \frac{\mathrm{d} k_{0}}{\mathrm{~d} z} B=0 .
\end{aligned}
$$

This separation is accomplished by setting equal to zero the various terms in $x^{2}, y^{2}, x$, and $y$. The significance of the $Q$ parameters is contained in the relation

$$
Q_{x}(z)=\frac{k_{0}(z)}{R_{x}(z)}-i \frac{2}{w_{x}^{2}(z)},
$$

where $R_{x}$ and $w_{x}$ are, respectively, the radius of curvature of the phase fronts and the $1 / e$ amplitude spot size in the $x$ direction. The ratio $d_{x a}=-S_{x i} / Q_{x i}$ is the displacement in the $x$ direction of the amplitude center of the Gaussian part of the beam, and the ratio $d_{x p}$ $=-S_{x r} / Q_{x r}$ is the displacement in the $x$ direction of the phase center of the beam. ${ }^{11}$ Here the subscripts $i$ and $r$ 
denote, respectively, the imaginary and real parts of the parameters $Q_{x}$ and $S_{x}$, and similar relations apply to the functions $Q_{y}$ and $S_{y}$.

Thus far we have obtained a general set of off-axis Gaussian beams governed by Eqs. (6)-(9), and the solutions of these equations are known for $z$-independent media. ${ }^{11}$ Additional amplitude and phase variations can be found as solutions of Eq. (10). In the special case that $B(x, y, z)$ is independent of $x$ and $y$, the solutions correspond to the fundamental Gaussian beam. If $B(x, y, z)$ is not independent of $x$ and $y$, Eq. (10) can still be solved exactly. In fact, several different solution sets are possible, and most previous solutions have included various on-axis and off-axis Hermite-Gaussian and LaguerreGaussian beams. The solutions reported here involve general off-axis sinusoidal-Gaussian beams of complex argument.

To maintain as much generality as possible, one is led to modify Eq. (10) with the following changes of variables $^{12}$ :

$$
\begin{aligned}
& x^{\prime}=a_{x}(z) x+b_{x}(z), \\
& y^{\prime}=a_{y}(z) y+b_{y}(z), \\
& z^{\prime}=z,
\end{aligned}
$$

where $a_{x}(z), b_{x}(z), a_{y}(z)$, and $b_{y}(z)$ are as yet unspecified functions of $z$. With these substitutions, Eq. (10) becomes

$$
\begin{aligned}
& a_{x}{ }^{2} \frac{\partial^{2} B}{\partial x^{\prime 2}}-2\left[i a_{x^{\prime}} S_{x}+i\left(x^{\prime}-b_{x}\right) Q_{x}\right. \\
& \left.+i k_{0} \frac{\left(x^{\prime}-b_{x}\right)}{a_{x}} \frac{\mathrm{d} a_{x}}{\mathrm{~d} z^{\prime}}+i k_{0} \frac{\mathrm{d} b_{x}}{\mathrm{~d} z^{\prime}}\right] \frac{\partial B}{\partial x^{\prime}} \\
& +a_{y}{ }^{2} \frac{\partial^{2} B}{\partial y^{\prime 2}}-2\left[i a_{y} S_{y}+i\left(y^{\prime}-b_{y}\right) Q_{y}\right. \\
& \left.+i k_{0} \frac{\left(y^{\prime}-b_{y}\right)}{a_{y}} \frac{\mathrm{d} a_{y}}{\mathrm{~d} z^{\prime}}+i k_{0} \frac{\mathrm{d} b_{y}}{\mathrm{~d} z^{\prime}}\right] \frac{\partial B}{\partial y^{\prime}} \\
& -\left(S_{x}{ }^{2}+S_{y}{ }^{2}\right) B-i\left(Q_{x}+Q_{y}\right) B \\
& \quad-2 i k_{0} \frac{\partial B}{\partial z^{\prime}}-i \frac{\mathrm{d} k_{0}}{\mathrm{~d} z^{\prime}} B=0
\end{aligned}
$$

The substitution

$$
B\left(x^{\prime}, y^{\prime}, z^{\prime}\right)=C\left(x^{\prime}, y^{\prime}, z^{\prime}\right) \exp \left[-i P\left(z^{\prime}\right)\right]
$$

in Eq. (15) makes possible the arbitrary separation

$$
\begin{aligned}
\frac{\mathrm{d} P}{\mathrm{~d} z^{\prime}}= & -\frac{1}{2 k_{0}}\left[\left(S_{x}^{2}+S_{y}^{2}\right)+i\left(Q_{x}+Q_{y}\right)\right. \\
& \left.+\left(\gamma_{x}^{2} a_{x}^{2}+\gamma_{y}^{2} a_{y}^{2}\right)+i \frac{\mathrm{d} k_{0}}{\mathrm{~d} z^{\prime}}\right]
\end{aligned}
$$

$$
\begin{gathered}
a_{x}^{2} \frac{\partial^{2} C}{\partial x^{\prime 2}}-2\left[i a_{x} S_{x}+i\left(x^{\prime}-b_{x}\right) Q_{x}+i k_{0} \frac{\left(x^{\prime}-b_{x}\right)}{a_{x}} \frac{\mathrm{d} a_{x}}{\mathrm{~d} z^{\prime}}\right. \\
\left.+i k_{0} \frac{\mathrm{d} b_{x}}{\mathrm{~d} z^{\prime}}\right] \frac{\partial C}{\partial x^{\prime}}+a_{y}{ }^{2} \frac{\partial^{2} C}{\partial y^{\prime 2}} \\
-2\left[i a_{y} S_{y}+i\left(y^{\prime}-b_{y}\right) Q_{y}+i k_{0} \frac{\left(y^{\prime}-b_{y}\right)}{a_{y}} \frac{\mathrm{d} a_{y}}{\mathrm{~d} z^{\prime}}\right. \\
\left.+i k_{0} \frac{\left.\mathrm{d} b_{y}\right]}{\mathrm{d} z^{\prime}}\right] \frac{\partial C}{\partial y^{\prime}}-2 i k_{0} \frac{\partial C}{\partial z^{\prime}} \\
\quad+\left(\gamma_{x}{ }^{2} a_{x}{ }^{2}+\gamma_{y}{ }^{2} a_{y}{ }^{2}\right) C=0
\end{gathered}
$$

where $P\left(z^{\prime}\right)$ is a phase parameter and $\gamma_{x}$ and $\gamma_{y}$ are separation constants. Equation (18) may be reduced to equations for sinusoidal functions if the quantities in brackets are set equal to zero. Then this equation initially becomes

$$
\begin{aligned}
a_{x}^{2}\left(\frac{\partial^{2} C}{\partial x^{\prime 2}}+\gamma_{x}^{2} C\right)+a_{y}{ }^{2}\left(\frac{\partial^{2} C}{\partial y^{\prime 2}}+\right. & \left.\gamma_{y}^{2} C\right) \\
& -2 i k_{0} \frac{\partial C}{\partial z^{\prime}}=0 .
\end{aligned}
$$

The corresponding constraints on $a_{x}\left(z^{\prime}\right)$ and $b_{x}\left(z^{\prime}\right)$ that arise from equating separately the terms in the first bracketed quantity of Eq. (18) that multiply $x^{\prime}$ and those that do not may be written

$$
\begin{array}{r}
Q_{x}+\frac{k_{0}}{a_{x}} \frac{\mathrm{d} a_{x}}{\mathrm{~d} z^{\prime}}=0, \\
a_{x} S_{x}+k_{0} \frac{\mathrm{d} b_{x}}{\mathrm{~d} z^{\prime}}=0,
\end{array}
$$

where Eq. (20) has also been used in simplifying Eq. (21). Similar equations are obtained for $a_{y}\left(z^{\prime}\right)$ and $b_{y}\left(z^{\prime}\right)$.

The product function

$$
C\left(x^{\prime}, y^{\prime}, z^{\prime}\right)=X\left(x^{\prime}\right) Y\left(y^{\prime}\right)
$$

satisfies Eq. (19) provided that $X$ and $Y$ are solutions of the sine function differential equations

$$
\begin{aligned}
& \frac{\partial^{2} X}{\partial x^{\prime 2}}+\gamma_{x}^{2} X=0 \\
& \frac{\partial^{2} Y}{\partial y^{\prime 2}}+\gamma_{y}^{2} Y=0
\end{aligned}
$$

Thus the general solution for the propagation of optical beams in complex $z$-dependent lenslike media can be expressed in terms of sinusoidal-Gaussian functions of complex argument. The previous results can be collected together and written explicitly as 


$$
\begin{aligned}
E_{x}{ }^{\prime}(x, y, z)= & E_{x 0}{ }^{\prime} \exp \left\{-i\left[\int_{0}^{z} k_{0}\left(z^{\prime}\right) \mathrm{d} z^{\prime}+\frac{Q_{x}(z) x^{2}}{2}+\frac{Q_{y}(z) y^{2}}{2}+S_{x}(z) x+S_{y}(z) y+P(z)\right]\right\} \\
& \times\left(\begin{array}{c}
\sin \left\{\gamma_{x}\left[a_{x}(z) x+b_{x}(z)\right]\right\} \\
\cos \left\{\gamma_{x}\left[a_{x}(z) x+b_{x}(z)\right]\right\} \\
\sinh \left\{\gamma_{x}^{\prime}\left[a_{x}(z) x+b_{x}(z)\right]\right\} \\
\cosh \left\{\gamma_{x}^{\prime}\left[a_{x}(z) x+b_{x}(z)\right]\right\}
\end{array}\right)\left(\begin{array}{c}
\sin \left\{\gamma_{y}\left[a_{y}(z) y+b_{y}(z)\right]\right\} \\
\cos \left\{\gamma_{y}\left[a_{y}(z) y+b_{y}(z)\right]\right\} \\
\sinh \left\{\gamma_{y}{ }^{\prime}\left[a_{y}(z) y+b_{y}(z)\right]\right\} \\
\cosh \left\{\gamma_{y}{ }^{\prime}\left[a_{y}(z) y+b_{y}(z)\right]\right\}
\end{array}\right), \quad(25)
\end{aligned}
$$

where $\gamma_{x}{ }^{\prime}=i \gamma_{x}, \gamma_{y}{ }^{\prime}=i \gamma_{y}$, and the terms in large parentheses are meant to suggest a possible superposition of the sine, cosine, hyperbolic sine, and hyperbolic cosine symmetries, which could also be represented as, for example, just the sine or sinh functions but with added phase terms. The separation constants $\gamma_{x}$ and $\gamma_{y}$ could
In this expression $R_{x}$ represents the radius of curvature of the phase fronts, $w_{x}$ is the spot size or $1 / e$ amplitude radius of the Gaussian amplitude distribution, $\lambda$ is the vacuum wavelength, and $n_{0}$ is the index of refraction. For a $z$-independent medium the $A B C D$ coefficients in $\mathrm{Eq}$. (26) are the elements of the matrix

$$
\left[\begin{array}{l}
A_{x} B_{x} \\
C_{x} D_{x}
\end{array}\right]=\left[\begin{array}{cc}
\cos \left[\left(k_{2 x} / k_{0}\right)^{1 / 2} z\right] & \left(k_{0} / k_{2 x}\right)^{1 / 2} \sin \left[\left(k_{2 x} / k_{0}\right)^{1 / 2} z\right] \\
-\left(k_{2 x} / k_{0}\right)^{1 / 2} \sin \left[\left(k_{2 x} / k_{0}\right)^{1 / 2} z\right] & \cos \left[\left(k_{2 x} / k_{0}\right)^{1 / 2} z\right]
\end{array}\right] .
$$

now be absorbed into their associated $a$ and $b$ coefficients, or, equivalently, they can be set equal to unity. Equation (25) is our general form for the sinusoidal-Gaussian beams of complex argument in complex lenslike media.

\section{SOLUTION OF THE BEAM EQUATIONS}

In the previous section we derived a set of beam solutions that can describe the spatial distribution of electromagnetic waves as they propagate in general complex lenslike media. In this process the partial differential wave equation has been reduced to a set of ordinary differential equations. Our solutions will not be complete until these secondary beam-parameter equations have actually been solved. Thus it is now necessary to solve the coupled ordinary first-order differential equations given above as Eqs. (6)-(9), (17), (20), and (21).

Although many specific solutions exist for the beam parameter Eqs. (6) and (7), no completely general analytic solution for $Q_{x}$ and $Q_{y}$ is available for arbitrary $z$ dependences of the wave-number coefficients $k_{0}, k_{1}$, and $k_{2}$. Numerical solutions are of course always possible, and it will be seen that all of the other coefficients in the mode expressions can be expressed in terms of $Q_{x}$ and $Q_{y}$. The solutions to Eq. (6) can be written in the well-known form $^{3}$

$$
\frac{Q_{x 2}}{k_{0}}=\frac{1}{q_{x 2}}=\frac{C_{x}+D_{x} / q_{x 1}}{A_{x}+B_{x} / q_{x 1}},
$$

and this result is also valid for a wide variety of other optical elements. We will be using the standard low-gainper-wavelength form of the beam parameter ${ }^{25}$ :

$$
\frac{1}{q_{x}}=\frac{1}{R_{x}}-\frac{i \lambda}{n_{0} \pi w_{x}^{2}} .
$$

Similar solutions are obtained for Eq. (7). Equation (26) was referred to by Kogelnik as the $A B C D$ law $^{26}$ but has also otherwise been called the Kogelnik transformation.

Equation (26) gives the $z$ dependence of the beam parameter $Q_{x}$, and this result can now be substituted into Eq. (8) for the complex displacement parameter $S_{x}$. The resulting equation can be integrated explicitly. For simplicity we will make the specific choice that the medium is aligned with the $z$ axis $\left(k_{1 x}=k_{1 y}=0\right)$, and then the displacement parameter for the $x$ direction is governed by the $A B$ law $^{27}$

$$
S_{x 2}=\frac{S_{x 1}}{A_{x}+B_{x} / q_{x 1}}
$$

with a similar equation for the displacement parameter for the $y$ direction $S_{y 2}$.

In the present limit of an aligned medium, Eq. (20) for the parameter $a_{x}(z)$ is of the same form as Eq. (8) for the displacement parameter $S_{x}(z)$. Therefore the parameter $a_{x}(z)$ is also governed by an $A B$ law,

$$
a_{x 2}=\frac{a_{x 1}}{A_{x}+B_{x} / q_{x 1}}
$$

with a similar equation for $a_{y 2}$. With the results given above, Eq. (21) for the parameter $b_{x}(z)$ can be written as

$$
\frac{\mathrm{d} b_{x}}{\mathrm{~d} z}=-\frac{1}{k_{0}} a_{x}(z) S_{x}(z)=-\frac{1}{k_{0}} \frac{a_{x 1} S_{x 1}}{\left\{\cos \left[\left(k_{2 x} / k_{0}\right)^{1 / 2} z\right]+\left(k_{0} / k_{2 x}\right)^{1 / 2} \sin \left[\left(k_{2 x} / k_{0}\right)^{1 / 2} z\right] / q_{x 1}\right\}^{2}}
$$


This equation can be integrated, and the result is

$b_{x}(z)=b_{x 1}-\frac{a_{x 1} S_{x 1}}{k_{0}} \frac{\left(k_{0} / k_{2 x}\right)^{1 / 2} \sin \left[\left(k_{2 x} / k_{0}\right)^{1 / 2} z\right]}{\cos \left[\left(k_{2 x} / k_{0}\right)^{1 / 2} z\right]+\left(k_{0} / k_{2 x}\right)^{1 / 2} \sin \left[\left(k_{2 x} / k_{0}\right)^{1 / 2} z\right] / q_{x 1}}$,

or

$$
b_{x 2}=b_{x 1}-\frac{a_{x 1} S_{x 1}}{k_{0}} \frac{B_{x}}{A_{x}+B_{x} / q_{x 1}} .
$$

A similar result is obtained for $b_{y}(z)$.

The phase parameter follows from Eq. (17), and for $z$ independent media this equation is

$$
\begin{aligned}
\frac{\mathrm{d} P(z)}{\mathrm{d} z}= & -\frac{1}{2 k_{0}}\left[\left(S_{x}{ }^{2}+S_{y}{ }^{2}\right)+i\left(Q_{x}+Q_{y}\right)\right. \\
& \left.+\left(\gamma_{x}^{2} a_{x}{ }^{2}+\gamma_{y}{ }^{2} a_{y}{ }^{2}\right)\right] .
\end{aligned}
$$

With the same methods as before, this equation can be integrated. The result is

$$
\begin{aligned}
P_{2}= & P_{1}-\frac{i}{2}\left[\ln \left(A_{x}+B_{x} / q_{x 1}\right)+\ln \left(A_{y}+B_{y} / q_{y 1}\right)\right] \\
& -\frac{1}{2 k_{0}}\left[\frac{\left(S_{x 1}{ }^{2}+\gamma_{x}^{2} a_{x 1}^{2}\right) B_{x}}{A_{x}+B_{x} / q_{x 1}}\right. \\
& \left.+\frac{\left(S_{y 1}{ }^{2}+\gamma_{y}^{2} a_{y 1}{ }^{2}\right) B_{y}}{A_{y}+B_{y} / q_{y 1}}\right]
\end{aligned}
$$

which can be checked by differentiation. When the various parameter formulas discussed here are introduced into Eq. (25), one has a complete description of the propagation of sinusoidal-Gaussian beams in aligned complex lenslike media. These results also apply to lenses, retroreflectors, and the many other elements that can be represented by $A B C D$ matrices. Misaligned and displaced media are most easily treated by using a $3 \times 3$ matrix formulation..$^{24}$

\section{PROPAGATION IN FREE SPACE}

As an example of the previous results, we will now consider in detail the important special case of beam propagation in free space. The general propagation matrix given in Eq. (28) reduces for a uniform medium $\left(k_{2 x}\right.$ $=0$ ) to the simple form

$$
\left[\begin{array}{ll}
A_{x} & B_{x} \\
C_{x} & D_{x}
\end{array}\right]=\left[\begin{array}{ll}
1 & z \\
0 & 1
\end{array}\right]
$$

and if the medium is free space the parameter $k_{0}$ has the real value $2 \pi / \lambda$. With use of Eq. (36) the various parameter equations given above as Eqs. (26), (29), (30), (33), and (35) reduce to

$$
\begin{aligned}
\frac{1}{q_{x 2}} & =\frac{1 / q_{x 1}}{1+z / q_{x 1}}, \\
S_{x 2} & =\frac{S_{x 1}}{1+z / q_{x 1}},
\end{aligned}
$$

$$
\begin{aligned}
a_{x 2}= & \frac{a_{x 1}}{1+z / q_{x 1}} \\
b_{x 2}= & b_{x 1}-\frac{a_{x 1} S_{x 1} z / k_{0}}{1+z / q_{x 1}} \\
P_{2}= & P_{1}-\frac{i}{2}\left[\ln \left(1+z / q_{x 1}\right)+\ln \left(1+z / q_{y 1}\right)\right] \\
& -\frac{z}{2 k_{0}}\left(\frac{S_{x 1}{ }^{2}+\gamma_{x}^{2} a_{x 1}{ }^{2}}{1+z / q_{x 1}}+\frac{S_{y 1}{ }^{2}+\gamma_{y}{ }^{2} a_{y 1}{ }^{2}}{1+z / q_{y 1}}\right) .
\end{aligned}
$$

As a further specialization it will be assumed that both the Gaussian and sinusoidal factors of the beam remain on the $z$ axis $\left(S_{x 1}=S_{y 1}=b_{x 1}=b_{y 1}=0\right)$. Furthermore, it will be assumed that the beam is so wide in the $y$ direction $\left(1 / q_{y 1} \Rightarrow 0, \gamma_{y} a_{y 1} \Rightarrow 0\right)$ that it can be considered a simple slab geometry configuration. In these limits Eq. (25) governing the field can be written

$$
\begin{aligned}
E_{x}{ }^{\prime}(x, z)= & E_{x 0}{ }^{\prime} \exp \left\{-i\left[k_{0} z+\frac{k_{0} x^{2}}{2 q_{x}(z)}+P(z)\right]\right\} \\
& \times \sin \left[\gamma_{x} a_{x}(z) x\right],
\end{aligned}
$$

where, to be specific, only the sine form of the beam is shown. The remaining equations governing the parameters of the beam include Eqs. (37), (39), and the following simplified form of Eq. (41):

$$
P_{2}=P_{1}-\frac{i}{2} \ln \left(1+z / q_{x 1}\right)-\frac{z}{2 k_{0}} \frac{\gamma_{x}^{2} a_{x 1}^{2}}{1+z / q_{x 1}} .
$$

Equations (27) and (37) are familiar in Gaussian beam studies. If the spot size and phase front curvatures are referenced to their values at the waist of the Gaussian factor of the beam, these equations yield the standard results

$$
\begin{aligned}
& w_{x 2}=w_{x 0}\left[1+\left(z / z_{0}\right)^{2}\right]^{1 / 2}, \\
& R_{x 2}=z\left[1+\left(z_{0} / z\right)^{2}\right],
\end{aligned}
$$

where $z_{0}=\pi w_{x 0}{ }^{2} / \lambda$ is the Rayleigh length and $w_{x 0}$ is the spot size at the waist. To be still more specific, while emphasizing the initial form of the beam as a sine-Gaussian, it will be assumed that the parameter $a_{x 1}$ at the beam waist has the real value $a_{x 0}$. In this case the real and imaginary parts of $a_{x 2}$ in Eq. (39) can be written

$$
\begin{aligned}
& a_{x 2 r}=\frac{a_{x 0}}{1+\left(z / z_{0}\right)^{2}}, \\
& a_{x 2 i}=\frac{a_{x 0}\left(z / z_{0}\right)}{1+\left(z / z_{0}\right)^{2}} .
\end{aligned}
$$

From Eq. (43) the phase parameter can be separated into its real and imaginary parts according to 


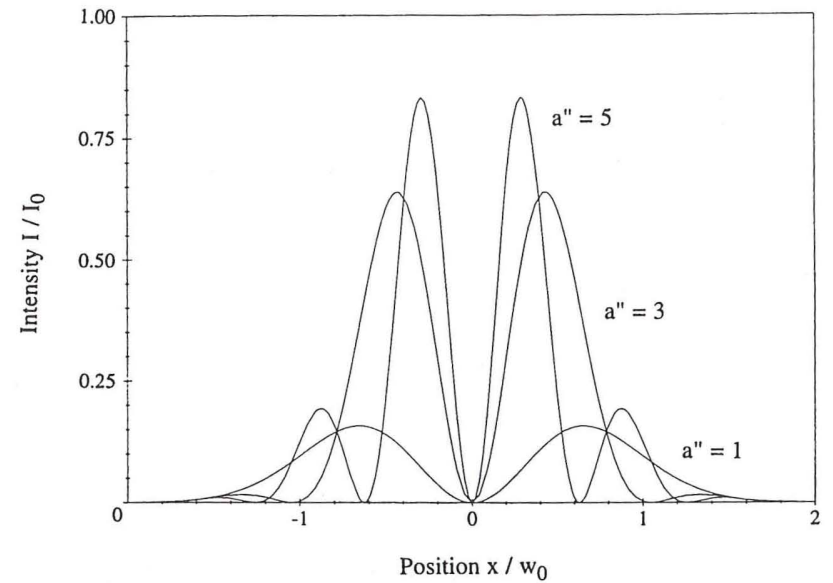

Fig. 1. Transverse intensity profiles of a sine-Gaussian beam at the reference plane $z^{\prime \prime}=0$ for the normalized modal parameter values $a^{\prime \prime}=1,3$, and 5 . For small values of this parameter the intensity distribution approaches that of a first-order HermiteGaussian beam mode.

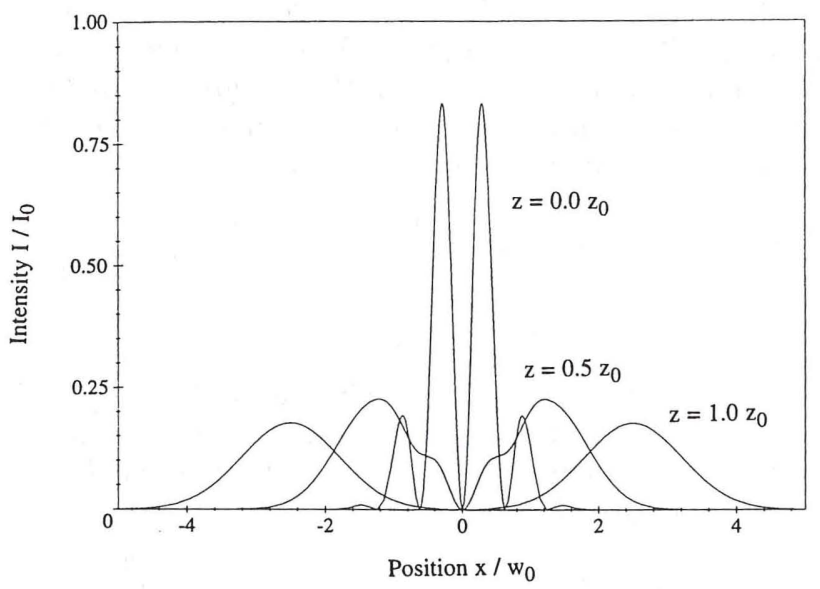

Fig. 2. Transverse intensity distribution of a sine-Gaussian beam with $a^{\prime \prime}=5$ for the normalized propagation distance values $z^{\prime \prime}=0.0,0.5,1.0$. For large values of the propagation distance the intensity distribution evolves into that of a sinhGaussian beam mode.

$$
\begin{aligned}
& P_{2 r}=-\frac{1}{2} \tan ^{-1}\left(z / z_{0}\right)-\frac{z \gamma_{x}^{2} a_{x 0}{ }^{2} /\left(2 k_{0}\right)}{1+\left(z / z_{0}\right)^{2}}, \\
& P_{2 i}=-\frac{1}{4} \ln \left[1+\left(z / z_{0}\right)^{2}\right]-\frac{z \gamma_{x}^{2} a_{x 0}{ }^{2}\left(z / z_{0}\right) /\left(2 k_{0}\right)}{1+\left(z / z_{0}\right)^{2}},
\end{aligned}
$$

where the initial phase has been set to zero.

The intensity is proportional to the product of the complex field amplitude $E_{x}{ }^{\prime}$ and its conjugate, and from Eqs. (42), (44)-(49) the intensity can be written as
Equation (50) is our final result for the intensity distribution of a pure sine-Gaussian beam propagating from a uniform-phase waist in free space. A similar result is obtained for a pure cosine-Gaussian beam.

The implications of Eq. (50) are not especially easy to visualize, so we have plotted some representative intensity profiles. As a first step it is helpful to introduce the normalized transverse coordinate $x^{\prime \prime}=x / w_{x 0}$, the normalized longitudinal coordinate $z^{\prime \prime}=z / z_{0}$, and the normalized parameter $a^{\prime \prime}=\gamma_{x} a_{x 0} w_{x 0}$. With these substitutions Eq. (50) reduces to the more compact form

$$
\begin{aligned}
I\left(x^{\prime \prime}, z^{\prime \prime}\right)= & \frac{I_{0}}{\left(1+z^{\prime \prime 2}\right)^{1 / 2}} \exp \left(-\frac{2 x^{\prime \prime 2}+a^{\prime \prime 2} z^{\prime \prime 2} / 2}{1+z^{\prime \prime 2}}\right) \\
& \times\left[\cosh \left(\frac{2 a^{\prime \prime} x^{\prime \prime} z^{\prime \prime}}{1+z^{\prime \prime 2}}\right)-\cos \left(\frac{2 a^{\prime \prime} x^{\prime \prime}}{1+z^{\prime \prime}}\right)\right] / 2 .
\end{aligned}
$$

We will look first at possible forms that this intensity profile may take at the reference plane $z^{\prime \prime}=0$. From Eq. (51) the intensity at this plane simplifies to

$$
\begin{aligned}
I\left(x^{\prime \prime}, z^{\prime \prime}=0\right) & =I_{0} \exp \left(-2 x^{\prime \prime 2}\right)\left[1-\cos \left(2 a^{\prime \prime} x^{\prime \prime}\right)\right] / 2 \\
& =I_{0} \exp \left(-2 x^{\prime \prime 2}\right) \sin ^{2}\left(a^{\prime \prime} x^{\prime \prime}\right) .
\end{aligned}
$$

Figure 1 shows some typical transverse intensity profiles of a sine-Gaussian beam based on Eq. (52), for various values of the normalized parameter $a^{\prime \prime}$. For small values of this parameter, Eq. (52) reduces to

$$
I\left(x^{\prime \prime}, z^{\prime \prime}=0\right) \approx I_{0} a^{\prime \prime 2} x^{\prime 2} \exp \left(-2 x^{\prime \prime 2}\right),
$$

which is the intensity distribution of a first-order Hermite-Gaussian beam. Under the same conditions the intensity distribution of a cosine-Gaussian beam would approach that of the fundamental Gaussian beam.

It is also of interest to consider the transverse intensity distribution of a sine-Gaussian beam at various values of the propagation distance $z^{\prime \prime}$, and plots of this variation are given in Fig. 2. For large values of $z^{\prime \prime}$, Eq. (51) simplifies to

$$
\begin{aligned}
I\left(x^{\prime \prime}, z^{\prime \prime} \Rightarrow \infty\right)= & I_{0} z^{\prime \prime-1} \exp \left[-\left(2 x^{\prime \prime 2} / z^{\prime \prime 2}+a^{\prime \prime 2} / 2\right)\right] \\
& \times\left[\cosh \left(2 a^{\prime \prime} x^{\prime \prime} / z^{\prime \prime}\right)-1\right] / 2 \\
= & I_{0} z^{\prime \prime-1} \exp \left[-\left(2 x^{\prime \prime} / z^{\prime \prime 2}+a^{\prime \prime 2} / 2\right)\right] \\
& \times \sinh ^{2}\left(a^{\prime \prime} x^{\prime \prime} / z^{\prime \prime}\right) .
\end{aligned}
$$

Thus the sine-Gaussian beam evolves into a sinhGaussian beam as it propagates. In a similar way the cosine-Gaussian beam would evolve into a cosh-Gaussian beam.

The cosh-Gaussian beam in particular may have important applications in optimizing the efficiency of laser amplifiers. To illustrate this, we will note from the form of

$$
I(x, z)=\frac{I_{0}}{\left[1+\left(z / z_{0}\right)^{2}\right]^{1 / 2}} \exp \left[-\frac{2\left(x / w_{x 0}\right)^{2}+\left(z_{0} \gamma_{x}^{2} a_{x 0}{ }^{2} / k_{0}\right)\left(z / z_{0}\right)}{1+\left(z / z_{0}\right)^{2}}\right]\left\{\cosh \left[\frac{2 \gamma_{x} x a_{x 0}\left(z / z_{0}\right)}{1+\left(z / z_{0}\right)^{2}}\right]-\cos \left[\frac{2 \gamma_{x} x a_{x 0}}{1+\left(z / z_{0}\right)^{2}}\right]\right\} / 2 .
$$




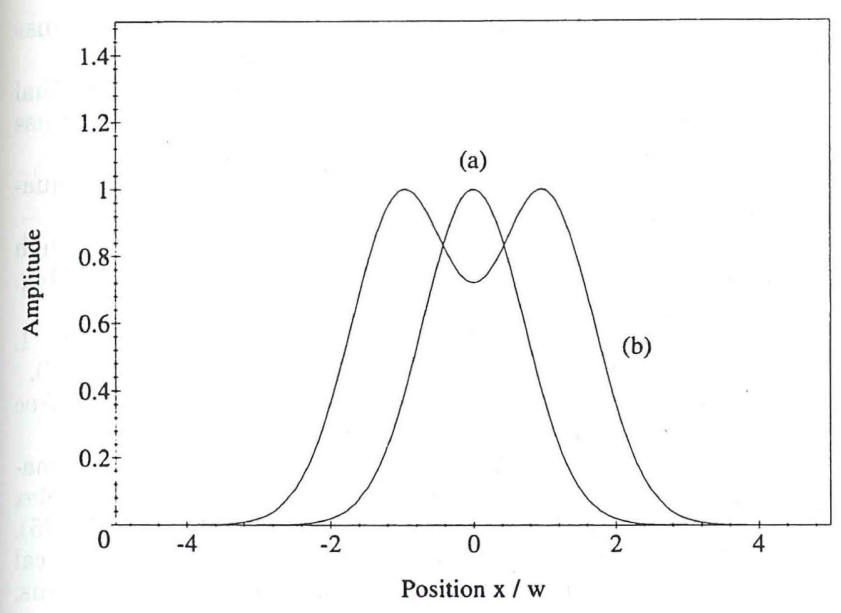

Fig. 3. Amplitude profiles from Eq. (55) for (a) a fundamental Gaussian beam and (b) a cosh-Gaussian beam. The horizontal variable for these plots is $x / w$, and the vertical variable is normalized to unity. The cosh scale width for part (b) is given by $a \times w=5$.

Eq. (42) that at any value of the position $z$ the transverse amplitude factors of a cosh-Gaussian beam are proportional to

$$
E_{x}{ }^{\prime}(x, z) \propto \cosh [a(z) x] \exp \left[-x^{2} / w(z)^{2}\right] .
$$

This amplitude profile is plotted in Fig. 3, where it is also compared with the more familiar Gaussian amplitude factor. It is clear from the figure that the cosh-Gaussian beam can be much squarer than other beam profiles, depending on the specific values of the width coefficients in the equation. Thus, these profiles can resemble closely the super-Gaussian field distributions that are known to be more efficient at extracting energy from an amplifying medium. $^{28}$

\section{DISCUSSION}

Sinusoidal-Gaussian beams have been obtained here for the propagation of electromagnetic waves in free space and in complex media. In the limit of large Gaussian beam spot size the Gaussian beam factor becomes unimportant, and the field distribution reduces to the conventional modes of a rectangular waveguide. In the opposite limit that the period of the sinusoidal factors is large compared with the width of the Gaussian factor, the beam takes the form of the familiar fundamental Gaussian beam (or sometimes the first-order Hermite-Gaussian beam). A different but similar class of beams involves hyperbolic-sinusoidal-Gaussian functions.

None of these classes of beams is difficult to obtain in the laboratory. The easiest method would be to have an ordinary Gaussian beam be incident on an appropriate transmission or reflection aperture. For example, if a Gaussian beam is incident on an aperture having a cosh amplitude transmission function (at least over the dimensions of the beam), one immediately obtains a coshGaussian beam. Although the detailed amplitude and phase profiles of this beam will evolve with further propagation through free space or through an arbitrary $A B C D$ optical system, the general functional form of the beam will remain expressible as cosh-Gaussian. Such beams are of practical interest because of their potentially more efficient extraction of energy from conventional laser amplifiers.

Although the example just mentioned involved a hyperbolic sinusoidal-Gaussian beam, the nonhyperbolic sinusoidal-Gaussian beams are equally interesting. These beams could also be readily obtained by means of an appropriate transmission filter, and there are important applications for spatially modulated Gaussian laser beams. $^{29}$ Any thin optical element with a periodic transverse amplitude (or phase) transmission (or reflection) profile, such as a grating, would yield a transformation profile that could be represented as a Fourier series. Each of the resulting harmonics might be imagined to convert a portion of the input beam into a corresponding sinusoidal-Gaussian. However, since field reversals occur between neighboring areas of the desired sinusoidalGaussian beam, an ideal sinusoidal transmission filter would include a phase plate to modify both the amplitude and the phase of the incident Gaussian beam. The resulting sinusoidal-Gaussian beams could be propagated through conventional optical elements by using standard $A B C D$ matrix methods, and they might also be useful in transforming between the Gaussian free-space beams and the sinusoidal modes that commonly occur in waveguides.

To some extent, the Hermite-Gaussian and sinusoidalGaussian solutions may be considered to compete with each other as representations for propagating electromagnetic beams. However, if the input field is primarily sinusoidal-Gaussian in form, several Hermite-Gaussian solutions might be required to represent it, and vice versa. Thus the choice of field representation would probably be made on the basis of efficiency, and the beam solutions described here would provide the optical system designer with a sometimes welcome alternative. A more detailed discussion of applications of the sinusoidalGaussian beam solutions will be given elsewhere.

\section{ACKNOWLEDGMENT}

This research was supported in part by the National Science Foundation under grants ECS-9422572 and PHY9415583.

*Permanent address, Department of Electrical Engineering, Portland State University, P.O. Box 751, Portland, Oregon 97207-0751.

\section{REFERENCES}

1. G. Goubau and F. Schwering, "On the guided propagation of electromagnetic wave beams," IRE Trans. Antennas Propag. AP-9, 248-256 (1961).

2. G. D. Boyd and J. P. Gordon, "Confocal multimode resonator for millimeter through optical wavelength masers," Bell Syst. Tech. J. 40, 489-508 (1961).

3. H. Kogelnik, "On the propagation of Gaussian beams of light through lenslike media including those with a loss or gain profile," Appl. Opt. 4, 1562-1569 (1965).

4. L. W. Casperson and A. Yariv, "The Gaussian mode in optical resonators with a radial gain profile," Appl. Phys. Lett. 12, 355-357 (1968).

5. N. G. Vakhimov, "Open resonators with mirrors having variable reflection coefficients," Radio Eng. Electron. Phys. (USSR) 10, 1439-1446 (1965). 
6. H. Zucker, "Optical resonators with variable reflectivity mirrors," Bell Syst. Tech. J. 49, 2349-2376 (1970).

7. J. A. Arnaud, "Nonorthogonal optical waveguides and resonators," Bell Syst. Tech. J. 49, 2311-2348 (1970).

8. J. A. Arnaud, "Mode coupling in first order optics," J. Opt. Soc. Am. 61, 751-758 (1971).

9. A. E. Siegman, "Hermite-Gaussian functions of complex argument as optical-beam eigenfunctions," J. Opt. Soc. Am. 63, 1093-1094 (1973).

10. J. A. Arnaud, Beam and Fiber Optics (Academic, New York, 1976), especially Subsecs. 2.16, 2.17, and 4.18-4.20.

11. L. W. Casperson, "Gaussian light beams in inhomogeneous media,” Appl. Opt. 12, 2434-2441 (1973).

12. L. W. Casperson, "Beam modes in complex lenslike media and resonators," J. Opt. Soc. Am. 66, 1373-1379 (1976).

13. R. Pratesi and L. Ronchi, "Generalized Gaussian beams in free space,” J. Opt. Soc. Am. 67, 1274-1276 (1977).

14. M. Nazarathy, A. Hardy, and J. Shamir, "Generalized mode propagation in first-order optical systems with loss or gain," J. Opt. Soc. Am. 72, 1409-1420 (1982).

15. A. E. Siegman, Lasers (University Science, Mill Valley, California, 1986), especially Subsec. 20.5.

16. A. A. Tovar and L. W. Casperson, "Off-axis complexargument polynomial-Gaussian beams in optical systems," J. Opt. Soc. Am. A 8, 60-68 (1991).

17. C. J. R. Sheppard and T. Wilson, "Gaussian-beam theory of lenses with annular aperture," Microwaves Opt. Acoust. 2, 105-112 (1978).
18. F. Gori, G. Guattari, and C. Padovani, "Bessel-Gauss beams," Opt. Commun. 64, 491-495 (1987).

19. R. H. Jordan and D. G. Hall, "Free-space azimuthal paraxial wave equation: the azimuthal Bessel-Gauss beam solution," Opt. Lett. 19, 427-429 (1994).

20. D. G. Hall, "Vector-beam solutions of Maxwell's wave equation," Opt. Lett. 21, 9-11 (1996).

21. Lord Rayleigh, "On the passage of electric waves through tubes, or the vibrations of dielectric cylinders," Philos. Mag Ser. 5 43, 125-132 (1897).

22. J. E. Durnin, "Exact solutions for nondiffracting beams. I The scalar theory," J. Opt. Soc. Am. A 4, 651-654 (1987).

23. J. E. Durnin, J. J. Miceli, and J. H. Eberly, "Diffraction-free beams," Phys. Rev. Lett. 58, 1499-1501 (1987).

24. A. A. Tovar and L. W. Casperson, "Generalized beam matrices: Gaussian beam propagation in misaligned complex optical systems,” J. Opt. Soc. Am. A 12, 1522-1533 (1995).

25. A. A. Tovar and L. W. Casperson, "Gaussian beam optical systems with high gain or high loss media," IEEE Trans. Microwave Theory Tech. 43, 1857-1862 (1995).

26. H. Kogelnik, "Imaging of optical modes-resonators with internal lenses," Bell Syst. Tech. J. 44, 455-494 (1965).

27. L. W. Casperson, "Mode stability in lasers and periodic optical systems," IEEE J. Quantum Electron. QE-10, 629634 (1974)

28. See, for example, E. Sklar, "Fourier-transform ring laser," J. Opt. Soc. Am. A 1, 537-540 (1984).

29. L. W. Casperson, "Spatial modulation of Gaussian laser beams," Opt. Quantum Electron. 10, 483-493 (1978). 